CLINICAL STUDY

\title{
Safety, tolerability, and pharmacokinetics of a single dose of pasireotide long-acting release in healthy volunteers: a single-center Phase I study
}

\author{
Hartmut Dietrich, $\mathrm{Ke} \mathrm{Hu}^{1}$, Matthieu Ruffin ${ }^{2}$, Dongweon Song ${ }^{1}$, Emmanuel Bouillaud ${ }^{2}$, Yanfeng Wang ${ }^{1}$ and \\ Jens Hasskarl ${ }^{2}$ \\ ClinPharmCologne, Neurather Ring 1, D-51063 Köln, Germany, ${ }^{1}$ Novartis Pharmaceuticals, East Hanover, New Jersey, USA and ${ }^{2}$ Novartis Pharma AG, \\ Basel, Switzerland \\ (Correspondence should be addressed to H Dietrich; Email: hartmut.dietrich@clinpharmcologne.de)
}

\begin{abstract}
Objective: This study was conducted to evaluate the safety, tolerability, and pharmacokinetics (PKs) of different doses of a long-acting release (LAR) formulation of pasireotide in healthy subjects.

Design: Single-center, open-label, randomized Phase I study.

Methods: Twelve healthy male subjects received a single s.c. dose of pasireotide $300 \mu \mathrm{g}$ followed by a washout period of 7 days (or at least 5 days), before receiving an i.m. injection of pasireotide -LAR $40 \mathrm{mg}(n=5)$ or $60 \mathrm{mg}(n=7)$. Assessments included adverse events (AEs), PKs, and glucose, insulin, glucagon, and HbA1c levels.

Results: Pasireotide LAR showed an extended-release profile over 1 month with two concentration peaks observed 1 and around 20 days after injection. The area under curve exposure of pasireotide LAR was dose proportional when the dose levels were compared, and the bioavailability of the LAR relative to the s.c. formulation was complete. Administration of pasireotide LAR resulted in an increase in fasting and postprandial glucose levels; however, an attenuation of the hyperglycemic effect was observed after 15 days. The most frequently reported AEs were mild-to-moderate diarrhea, abdominal pain, and flatulence. Only gastrointestinal AEs and injection site reactions were suspected to be drug related.

Conclusions: Pasireotide LAR was generally well tolerated with mostly mild AEs at doses up to $60 \mathrm{mg}$ and showed a dose-proportional, extended-release profile in healthy subjects. Based on the favorable results of this study, further clinical development of pasireotide LAR is under way, which will give insight into the PKs, efficacy, and safety of pasireotide LAR in patient populations.
\end{abstract}

European Journal of Endocrinology 166 821-828

\section{Introduction}

Pasireotide (SOM230) is a cyclohexapeptide somatostatin analog with a molecular weight of $1047 \mathrm{kDa}$ and contains the structural elements [(2-aminoethyl)aminocarbonyl oxy]-L-proline, phenylglycine, and tyrosine (benzyl). Pasireotide was developed in a diaspartate form for s.c. administration (Fig. 1A) and in a pamoate form for i.m. injection as part of a long-acting release (LAR) formulation (Fig. 1B).

Pasireotide is a multireceptor-targeted somatostatin analog with high affinity for four of the five somatostatin receptor subtypes (sst; the greatest for sst $_{5}$ ) that regulate anterior pituitary hormone secretion (1). Compared with other somatostatin analogs such as octreotide and lanreotide, pasireotide binds with higher affinity to $\mathrm{sst}_{1}, \mathrm{sst}_{3}$, and $\mathrm{sst}_{5}$ and with slightly lower affinity to $s^{2}{ }_{2}(2)$. Given this unique receptor-binding profile, pasireotide may have enhanced potential in targeting pituitary adenomas, such as those seen in patients with Cushing's disease or acromegaly. The corticotroph pituitary adenomas that underlie Cushing's disease express multiple sst, but sst ${ }_{5}$ predominates (3). Activation of sst $_{5}$ inhibits ACTH secretion in vitro and is a potential therapeutic target for the treatment of Cushing's disease $(4,5,6)$. The rationale for the therapeutic applicability of pasireotide in acromegaly is also based on the differences in binding affinity and functional activity of pasireotide and octreotide. It has been suggested that in tumors that express sst other than $\mathrm{sst}_{2}$, pasireotide may have a stronger inhibitory effect on hormone secretion than octreotide.

In healthy volunteers, pasireotide is generally well tolerated when administered once or twice daily as a s.c. 

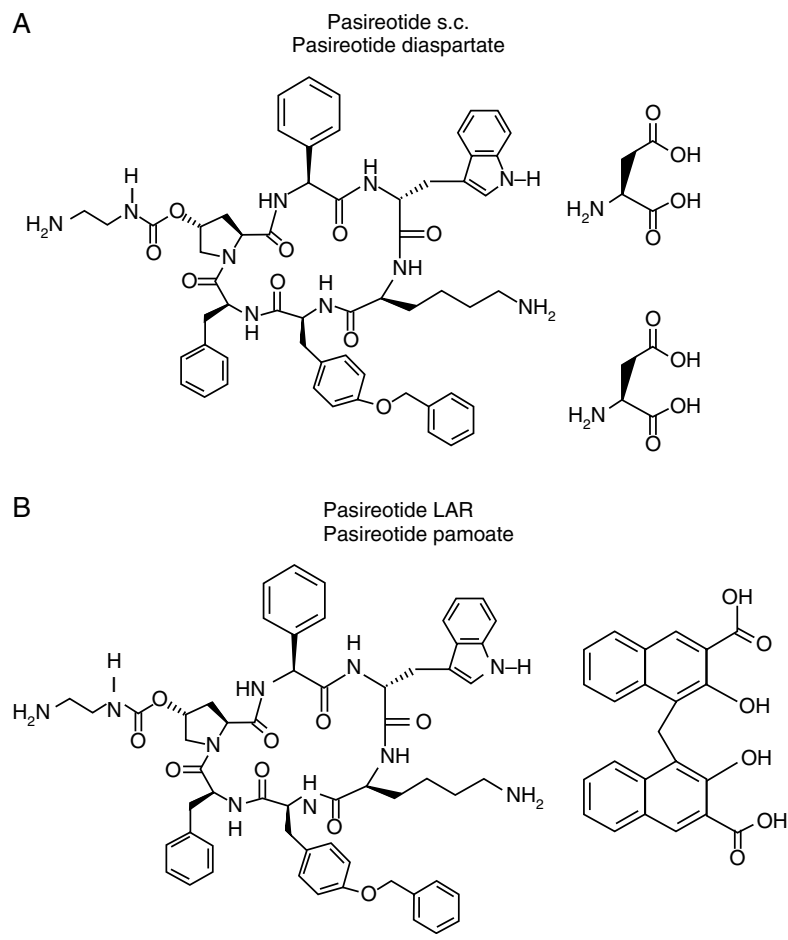

Figure 1 The chemical structures of pasireotide s.c. (A) and LAR (B).

injection and after daily infusion of doses up to $2050 \mu \mathrm{g}$ /day for 7 days $(7,8,9,10,11)$.

Phase II and III clinical studies have shown that twice-daily pasireotide s.c. is effective in controlling $\mathrm{GH}$ and insulin-like growth factor 1 (IGF1) levels and reducing pituitary tumor volume in patients with acromegaly $(12,13)$ and urinary free cortisol levels in patients with Cushing's disease $(14,15)$. These studies also showed that twice-daily pasireotide s.c. has a safety profile similar to that of other somatostatin analogs except for the degree of hyperglycemia, with no unexpected adverse events (AEs) reported.

For chronic disease treatment, twice-daily s.c. injections may not be convenient for patients, and development of a LAR formulation is warranted. The pasireotide LAR formulation was designed with biodegradable polymers to release the active drug over an extended period in order to facilitate once-monthly i.m. administration, which is similar in design to that of the octreotide LAR formulation. Furthermore, by controlling the drug release rate, pasireotide LAR is expected to produce less peak-to-trough fluctuation than pasireotide s.c., thereby potentially minimizing AEs such as nausea, vomiting, and hyperglycemia, which can be observed with immediate-release pasireotide s.c.

This paper presents the results of a single-center, open-label, Phase I study of the safety, tolerability, and pharmacokinetic (PK) profile of a single dose of pasireotide LAR in healthy subjects.

\section{Materials and methods}

\section{Subjects}

Healthy male subjects aged 18-40 years with a body mass index (BMI) of $20-30 \mathrm{~kg} / \mathrm{m}^{2}$, a body weight > $50 \mathrm{~kg}$, no history of relevant medical disorders, normal screening physical evaluation, and normal laboratory blood tests were enrolled in the study.

Subjects were excluded if they used any prescription drug or over-the-counter medication (except for paracetamol) 14 days before dosing, participated in any clinical investigation within 4 weeks prior, donated or lost $\geq 400 \mathrm{ml}$ of blood within 8 weeks before dosing, or had been diagnosed at any time as having either type 1 or type 2 diabetes mellitus or impaired glucose tolerance. A medical history of clinically significant electrocardiogram (ECG) abnormalities or a family history of a prolonged QT interval syndrome also resulted in exclusion. The study was approved by the independent ethics committee, research ethics board, or institutional review board and complied with the International Conference of Harmonization (ICH) Harmonized Tripartite Guidelines for Good Clinical Practice, the Declaration of Helsinki, and local laws. All subjects provided written informed consent.

\section{Study design}

This was a single-center, open-label, Phase I study. All subjects received a single dose of pasireotide $300 \mu \mathrm{g}$ s.c. in the abdominal wall followed by a washout period of 5-7 days before receiving an i.m. injection of pasireotide LAR into the left or right gluteal muscle. Subjects were consecutively allocated into a cohort; those in cohort 1 received pasireotide LAR $40 \mathrm{mg}$ and subjects in cohort 2 received pasireotide LAR $60 \mathrm{mg}$. All subjects were asked to report to the study site at $\sim 0800 \mathrm{~h}$ ( $1 \mathrm{~h}$ before dosing) for each visit in a fasted state for predose evaluations. The screening period was days -21 to -1 . Pasireotide $300 \mu \mathrm{g}$ s.c. was administered on study day 1 and pasireotide LAR (40 or $60 \mathrm{mg}$ ) was administered on study day 7. Day 70 was the study completion day.

\section{Objectives}

The primary objectives were to assess the safety, tolerability, and PK profile of different doses of pasireotide LAR. Secondary objectives were to estimate the bioavailability of pasireotide LAR at different dose levels relative to a single dose of an s.c. injection and to investigate the effects of pasireotide LAR on glucose, insulin, and glucagon levels.

\section{Assessments}

Clinical laboratory evaluations, ECG, vital signs, and physical examination results were recorded. ECG 
readings were taken on days $1,7,21$, and at end of study with a standard 12-lead ECG. The tracings were dated and signed by the person who made the interpretation and the same person evaluated the tracings throughout the study. Blood pressure and pulse rate were assessed before pasireotide s.c. or LAR injection on days 1 and 7 respectively, in the supine position and after standing for $3 \mathrm{~min}$. Assessments were also taken during the study screening period and on days 1,7 , and 21 at $0.5,1,2$, and $8 \mathrm{~h}$ after dosing. Single measures were taken on days 2 and 8 , every 2 days from days 9 to 35 , and on days 42, 49, 63, and 70 (end of study).

Blood samples for PK analysis were collected on days 1 and 7 at 0 (0.25h at day 1 only), $0.5,1,2,3,4,6,8$, 10 (12h at day 1 only), and $24 \mathrm{~h}$ at day 7 only. In addition, PK blood samples were collected every 2 days from study day 9 until study day 35 and on days 2, 42, 49 , and 63. Subjects returned to the study center on day 70 for study completion, evaluation, and final blood sample collection.

For measurement of fasting and postprandial glucose, insulin, and glucagon levels, subjects had 8-h assessments on 4 days during the study: screening visit (days -21 to -1 ), study day 1 (pasireotide $300 \mu$ g, s.c.), 7 (pasireotide LAR injection), and 21 (15 days after pasireotide LAR injection). During the 8-h assessments, subjects remained in the fasting state for the first $4 \mathrm{~h}$ and blood was collected at $0,0.5,1,2,3$, and $4 \mathrm{~h}$. After the 4-h collection time point, a standardized lunch was provided and blood was collected at 6 and $8 \mathrm{~h}$. HbAlc was measured at screening and at study end.

PK parameters analyzed after pasireotide LAR administration included the following: maximum concentrations observed in the first 3 days and from day 4 postdose ( $C_{\text {max,p1 }}$ and $C_{\text {max,p2 }}$ respectively), time to reach $C_{\text {max }}$ in the first 3 days and from day 4 postdose ( $t_{\text {max,p1 }}$ and $t_{\text {max,p2 }}$ respectively), area under the concentration-time curve from time zero to the last quantifiable concentration time point postdose $\left(\mathrm{AUC}_{\text {last }}\right), \mathrm{AUC}$ from zero to infinity $\left(\mathrm{AUC}_{\mathrm{inf}}\right)$, elimination half-life $\left(t_{1 / 2}\right)$, mean residence time (MRT), apparent total body clearance of drug from plasma (CL/F), apparent volume of distribution $\left(V_{\mathrm{z}} / F\right)$, and bioavailability of pasireotide LAR relative to pasireotide s.c. $\left(\mathrm{BA}_{\mathrm{LAR} / \mathrm{s.c} .}\right)$. Pasireotide LAR PK parameters $\left(\mathrm{AUC}_{\mathrm{inf}}\right.$, $\mathrm{AUC}_{\text {last }}, C_{\text {max,p1 }}$, and $C_{\text {max,p2 }}$ ) are presented as adjusted or corrected based on the actual dose of LAR administered, which was calculated based on the amount of drug left in the syringe after dosing using the following formula:

\section{Dose-adjusted parameter}

$$
=(\text { parameter/actual dose }) \times \text { planned dose. }
$$

Plasma concentrations were determined by a validated RIA with a lower limit of quantification of $150 \mathrm{pg} / \mathrm{ml}(0.15 \mathrm{ng} / \mathrm{ml}$; intra-assay and interassay precision: coefficient of variation $(\mathrm{CV} \%) \leq 30 \%$; accuracy: within $70-130 \%$ of the nominal concentration). Noncompartmental PK analysis was performed using WinNonlin software (version 5.2; Pharsight, Mountain View, CA, USA). Laboratory samples for PK bioanalysis were analyzed at Novartis Pharma AG.

AEs and serious AEs (SAEs) were recorded throughout the study period.

\section{Statistical analysis}

All subjects who received the planned doses of pasireotide LAR were included in the safety and tolerability analysis. The PK analysis population included all subjects who received the planned dose of pasireotide LAR and had adequate PK samples.

Descriptive statistics of $\mathrm{PK}$ parameters included mean, s.D., CV\%, minimum, maximum, geometric mean, and geometric mean $C V \%$. $t_{\max }$ was treated as a discrete time variable and, thus, median values and ranges were presented. The geometric mean and 90\% confidence interval (CI) of the bioavailability of pasireotide $\mathrm{LAR}$ relative to that of pasireotide s.c. was evaluated as follows: $\left(\mathrm{AUC}_{\mathrm{inf}, \mathrm{LAR}} / \mathrm{LAR}\right.$ dose $) /\left(\mathrm{AUC}_{\text {inf,s.c. }}{ }^{/-}\right.$ s.c. dose). RiaSmart was the statistical package used to acquire raw data and calculate concentrations.

One-way ANOVA with the Bonferroni post hoc test was used for statistical comparison for the AUC (both fasting and postprandial) for glucose, insulin, and glucagon at screening, study day 1 , and study day 15 . $P<0.05$ was considered significant. The paired $t$-test was used for statistical comparison for the AUC for glucose, insulin, and glucagon between the fasting and postprandial period.

\section{Results}

A total of 12 healthy subjects were enrolled to receive pasireotide LAR $40 \mathrm{mg}(n=5)$ or pasireotide LAR $60 \mathrm{mg}(n=7)$. Subject baseline demographics were similar between the two groups (Table 1). One subject in the pasireotide LAR $60 \mathrm{mg}$ cohort was excluded from the safety and PK analyses because the subject received only $\sim 15 \%$ of the planned dose due to a blockage in the syringe. Another subject in the pasireotide LAR $60 \mathrm{mg}$ group withdrew consent after receiving the LAR dose and discontinued. This subject did not have adequate PK samples to be included in the PK analysis. As such, five subjects per cohort were included in the PK analysis population.

\section{Pharmacokinetics}

Pasireotide LAR showed an extended-release profile following a single i.m. dose of 40 or $60 \mathrm{mg}$ in healthy volunteers. After administration, an initial increase in pasireotide concentration (burst) was observed with 
Table 1 Subject baseline demographics (safety population).

\begin{tabular}{lcc}
\hline & \multicolumn{2}{c}{ Pasireotide LAR } \\
\cline { 2 - 3 } & $40 \mathrm{mg}(n=5)$ & $60 \mathrm{mg}(n=6)$ \\
\hline Age (years), mean \pm s.D. & $28.6 \pm 2.61$ & $32.0 \pm 7.62$ \\
Sex - male, $n(\%)$ & $5(100)$ & $6(100)$ \\
Race-Caucasian, $n(\%)$ & $5(100)$ & $6(100)$ \\
Height $(\mathrm{cm})$, mean \pm s.D. & $181.0 \pm 6.82$ & $179.2 \pm 5.71$ \\
Weight $(\mathrm{kg})$, mean \pm s.D. & $81.1 \pm 4.91$ & $78.1 \pm 7.39$ \\
BMl $\left(\mathrm{kg} / \mathrm{m}^{2}\right)$, mean \pm s.D. & $24.8 \pm 2.27$ & $24.3 \pm 2.03$ \\
\hline
\end{tabular}

BMI, body mass index.

a peak at $10-24 \mathrm{~h}$ followed by a decrease from days 1 to 7. The slow-release phase then followed, characterized by a peak at around day 20 (pasireotide LAR $40 \mathrm{mg}$ : $t_{\text {max,p2 }} 18$ days; pasireotide LAR $60 \mathrm{mg}: t_{\text {max,p2 }} 22$ days) and a slow decline to the last sampling point (Fig. 2).

The exposure data of $C_{\text {max,p2 }}, \mathrm{AUC}_{\text {last }}$, and $\mathrm{AUC}_{\text {inf }}$ were approximately dose proportional for pasireotide LAR 40 and $60 \mathrm{mg}$. For the pasireotide LAR 40 and $60 \mathrm{mg}$ doses, $C_{\text {max.p1 }}$ values were $6.1 \pm 3.9$ and $5.5 \pm 1.2 \mathrm{ng} / \mathrm{ml}$ respectively; $C_{\text {max,p2 }}: 9.6 \pm 5.1$ and $15.8 \pm 3.3 \mathrm{ng} / \mathrm{ml}$; $\mathrm{AUC}_{\text {last }}: 4589 \pm 1153$ and $7516 \pm 705 \mathrm{ng} \mathrm{h} / \mathrm{ml} ; \mathrm{AUC}_{\mathrm{inf}}$ : $4959 \pm 1067$ and $7970 \pm 650 \mathrm{ng} \mathrm{h} / \mathrm{ml}$ respectively (Table 2). The bioavailability of pasireotide LAR relative to that of pasireotide s.c. was $148 \%$ (90\% CI: 130, 168) and $133 \%(90 \%$ CI: 110,155$)$ for the pasireotide LAR 40 and $60 \mathrm{mg}$ doses respectively. Individual subject plasma pasireotide concentration profiles for the pasireotide LAR 40 and $60 \mathrm{mg}$ doses are shown in Supplementary Figure 1, see section on supplementary data given at the end of this article.

\section{Safety and tolerability}

No SAEs or deaths occurred during the study. All subjects who received pasireotide LAR experienced AEs. The most frequently reported drug-related AEs were diarrhea, abdominal pain, injection site pain, and flatulence (Table 3), with the majority being mild or moderate in intensity.

No pasireotide LAR recipient showed QT prolongation during therapy, and no subject had either QT interval $\geq 480 \mathrm{~ms}$ or QTc interval $\geq 450 \mathrm{~ms}$ before or during the study. All subjects developed bradycardia during the study period with a maximum change from $60 \mathrm{bpm}$ (median; range: $51-73 \mathrm{bpm}$ ) at baseline to $55 \mathrm{bpm}$ (median; range: 46-68 bpm) at day 21 (15 days after pasireotide LAR injection). No subject developed clinically significant or symptomatic bradycardia, and all subjects had a heart rate similar to that exhibited at baseline and day 70 (end of study).

At screening, six subjects had a normal heart rate (>60 bpm; median $67 \mathrm{bpm}$; range 61-73 bpm) and six subjects had bradycardia ( $<60 \mathrm{bpm}$; median $57 \mathrm{bpm}$; range $51-58 \mathrm{bpm})$. Subjects with a normal heart rate at baseline developed maximum bradycardia at day 21 (median $55 \mathrm{bpm}$; range 46-68 bpm) that normalized by day 70 (median $63 \mathrm{bpm}$; range 50-81 bpm). Subjects with bradycardia at baseline remained bradycardic throughout the trial (at day 21: median $55 \mathrm{bpm}$, range 46-68 bpm; at day 70: median $59 \mathrm{bpm}$, range 50-65 bpm).

No clinically significant changes in liver function tests were found. No subjects displayed any significant abnormalities in the gallbladder ultrasounds recorded prestudy or at study completion.

\section{Glucose, insulin, and glucagon evaluations}

At screening, all 11 subjects had normal fasting blood glucose levels $(<100 \mathrm{mg} / \mathrm{dl}(<5.5 \mathrm{mmol} / \mathrm{l}))$. After injection of pasireotide LAR, nine subjects had a peak fasting blood glucose level that shifted to the next category $(100-125 \mathrm{mg} / \mathrm{dl}(5.6-6.9 \mathrm{mmol} / \mathrm{l}))$ and the remaining two subjects (one from each cohort) had fasting blood glucose levels $\geq 126 \mathrm{mg} / \mathrm{dl}(\geq 7.0 \mathrm{mmol} / \mathrm{l})$.

Pasireotide (either s.c. (on day 1) or LAR (on day 7)) resulted in increases in fasting glucose levels compared with baseline values (samples taken during the screening period). In addition, both pasireotide LAR 40 and $60 \mathrm{mg}$ recipients experienced elevated postprandial glucose levels, which appeared to be dose and concentration dependent (Supplementary Figure 2, see section on supplementary data given at the end of this article). Although postprandial values at day 21 (15 days after pasireotide LAR injection) remained higher than at baseline for both doses, the elevated fasting and postprandial blood glucose levels showed an attenuation compared with day 7 (Table 4). At the end of the study (70 days), fasting blood glucose levels had decreased to similar levels observed at baseline.

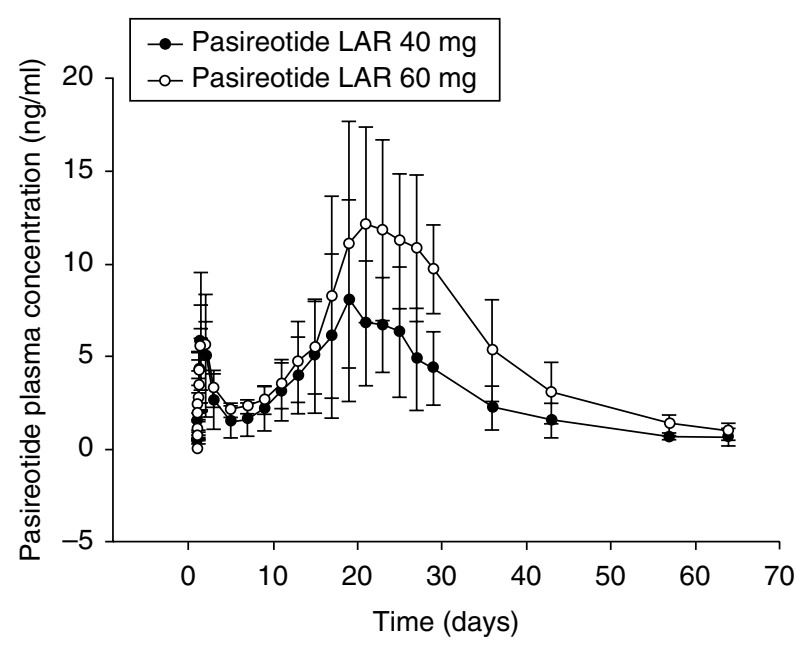

Figure 2 Mean (S.D.) plasma concentration vs time profiles of pasireotide in healthy volunteers following a single i.m. injection of pasireotide LAR 40 or $60 \mathrm{mg}$. In this figure, day 1 refers to the day on which pasireotide LAR was administered (study day 7 ). 
Table 2 Pharmacokinetic parameters of pasireotide LAR 40 and $60 \mathrm{mg}$. All parameters are presented as mean \pm S.D., except $t_{\max }$ data that are expressed as median (range).

\begin{tabular}{lcc}
\hline & \multicolumn{2}{c}{ Pasireotide LAR } \\
\cline { 2 - 3 } & $40 \mathrm{mg}(n=5)$ & $60 \mathrm{mg}\left(n=5^{\mathrm{a}}\right)$ \\
\hline$C_{\text {max }, \mathrm{p} 1}(\mathrm{ng} / \mathrm{ml})$ & $6.08 \pm 3.89$ & $5.52 \pm 1.20$ \\
$t_{\text {max }, \mathrm{p} 1}, \mathrm{~h}(\mathrm{range})$ & $10(8-10)$ & $24(10-25)$ \\
$C_{\text {max }, \mathrm{p} 2}(\mathrm{ng} / \mathrm{ml})$ & $9.60 \pm 5.13$ & $15.77 \pm 3.27$ \\
$t_{\text {max }}, \mathrm{h}(\mathrm{range})$ & $432(336-840)$ & $528(432-672)$ \\
$\mathrm{AUC}_{\text {last }}(\mathrm{ng} \mathrm{h} / \mathrm{ml})$ & $4589 \pm 1153$ & $7516 \pm 705$ \\
$\mathrm{AUC}_{\text {inf }}(\mathrm{ng} \mathrm{h} / \mathrm{ml})$ & $4959 \pm 1067$ & $7970 \pm 650$ \\
$t_{1 / 2}(\mathrm{~h})$ & $336 \pm 97$ & $316 \pm 76$ \\
$\mathrm{MRT}_{\text {last }}(\mathrm{h})$ & $588 \pm 119$ & $622 \pm 81$ \\
$\mathrm{MRT}_{\text {inf }}(\mathrm{h})$ & $699 \pm 211$ & $697 \pm 91$ \\
$\mathrm{CL} / \mathrm{F}(\mathrm{l} / \mathrm{h})$ & $8.5 \pm 2.4$ & $7.6 \pm 0.6$ \\
$V_{\mathrm{z}} / F(\mathrm{l})$ & $4196 \pm 1920$ & $3444 \pm 863$ \\
$\mathrm{BA}_{\text {LAR/s.c. }}$ & $1.48 \pm 0.15$ & $1.33 \pm 0.23$ \\
\hline
\end{tabular}

$C_{\text {max,p1 }}$, maximum concentration observed in the first 3 days; $t_{\max , \mathrm{p} 1}$, time to reach maximum concentration $\left(C_{\max }\right)$ in the first 3 days; $C_{\max , \mathrm{p} 2}$, maximum concentration observed from day 4 postdose; $t_{\max , \mathrm{p} 2}$, time to reach $C_{\max }$ from day 4 postdose; $A \cup C_{\text {last }}$, area under the concentration-time curve (AUC) from time zero to the last quantifiable concentration time point postdose (last) ; $\mathrm{AUC}_{\text {inf }}$, area under the concentration-time curve from zero to infinity; $t_{1 / 2}$, elimination half-life; MRT, mean residence time; $\mathrm{MRT}_{\text {last }}$, mean residence time determined as area under first moment curve (AUMC) $)_{\text {last }} / \mathrm{AUC}_{\text {last }}$ $\mathrm{MRT}_{\text {inf }}$, mean residence time determined as $A U M C_{\text {inf }} / \mathrm{AUC}_{\text {inf }} ; \mathrm{CL} / \mathrm{F}$, apparen total body clearance of drug from plasma; $V_{z} / F$, apparent volume of

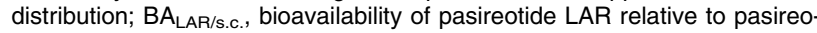
tide s.c.

${ }^{\mathrm{a} O n e}$ patient received $\sim 15 \%$ of the planned dose of LAR (10 mg instead of $60 \mathrm{mg}$ ) due to clogging inside the syringe and was excluded. Another subject received the LAR dose but withdrew consent and discontinued before providing adequate PK samples.

During the fasting period, pasireotide resulted in marked decreases in insulin AUC values compared with baseline values (Table 4), and the extent of the decrease appeared to be dose and concentration dependent (Supplementary Figure 2). After pasireotide LAR 40 and $60 \mathrm{mg}$ injection on day 7 , postprandial mean insulin AUC was higher than at baseline in both the 40 and $60 \mathrm{mg}$ cohorts. Fifteen days after pasireotide LAR injection, postprandial mean insulin AUC was lower than at baseline for both cohorts.

Decreases in glucagon AUC values during pasireotide treatment in the fasting period were smaller compared with decreases in insulin AUC values, but these were larger in the postprandial period. After pasireotide LAR 40 and $60 \mathrm{mg}$ injection, the mean glucagon AUC was lower than at baseline for both doses (Table 4). Fifteen days after pasireotide LAR injection, changes in mean glucagon AUC for both doses in the fasting state were more similar to those observed on the day of dosing. No changes in HbAlc levels were detected.

\section{Discussion}

Following a single i.m. injection in healthy subjects, pasireotide LAR showed a dose-proportional, extendedrelease profile suitable for monthly administration. A first peak (burst) in pasireotide concentration was observed on day 1 after administration of pasireotide LAR followed by a decrease in concentration from days 1 to 7 . A second slow-release peak with maximum concentrations around day 20 was observed, after which pasireotide plasma concentrations gradually decreased.

The plasma AUC ratio of pasireotide LAR relative to that of pasireotide s.c. was 148 and $133 \%$ for the 40 and $60 \mathrm{mg}$ doses respectively, suggesting that the bioavailability of the LAR formulation relative to the s.c. formulation was complete. The $>100 \%$ AUC ratio was probably due to an underestimation of the plasma AUC for the pasireotide $300 \mu \mathrm{g}$ s.c. dose as a result of the terminal phase falling below the limit of quantification of the bioanalytical assay.

In general, pasireotide LAR was well tolerated in this study of healthy volunteers. The majority of the observed AEs were typical of those expected with somatostatin analogs (16). The most frequently reported AEs were mild-to-moderate gastrointestinal disorders, predominantly diarrhea, which was sometimes associated with abdominal pain and flatulence. Adverse gastrointestinal events are commonly seen during the first few weeks of treatment with currently available somatostatin analogs and become less frequent during ongoing treatment (17). As only one dose of pasireotide LAR was administered to each subject in this study, the rate of AEs seen in this study may not represent the frequency of potential AEs during routine clinical use.

Administration of pasireotide LAR resulted in increases in fasting glucose levels and fasting glucose AUC values; elevated levels were seen 15 days after injection compared with baseline in both the fasting and the postprandial state. The hyperglycemic effect decreased over time, and by day 43, fasting blood glucose levels had returned to those observed during the screening visit. During the fasting period, there were also marked decreases in insulin AUC levels and small decreases in glucagon AUC levels relative to screening

Table 3 Adverse events suspected to be study-drug related.

\begin{tabular}{lcc}
\hline & \multicolumn{2}{c}{ Pasireotide LAR $(n(\%))$} \\
\cline { 2 - 3 } Adverse event & $40 \mathrm{mg}(n=5)$ & $60 \mathrm{mg}\left(n=6^{\mathrm{a}}\right)$ \\
\hline Diarrhea & $3(60)$ & $6(100)$ \\
Abdominal pain & $1(20)$ & $4(67)$ \\
Flatulence & $1(20)$ & $3(50)$ \\
Abdominal discomfort & 0 & $1(17)$ \\
Abnormal bowel sounds & - & $1(17)$ \\
Pale feces & $1(20)$ & $1(17)$ \\
Frequent bowel movements & 0 & $1(17)$ \\
Injection site pain & $4(80)$ & $1(17)$ \\
Dizziness & $1(20)$ & $1(17)$ \\
Headache & 0 & $1(17)$ \\
Abdominal distension & $1(20)$ & 0 \\
\hline
\end{tabular}

${ }^{a}$ One patient received $\sim 15 \%$ of the planned dose of LAR ( $10 \mathrm{mg}$ instead of $60 \mathrm{mg}$ ) due to clogging inside the syringe and was excluded. 


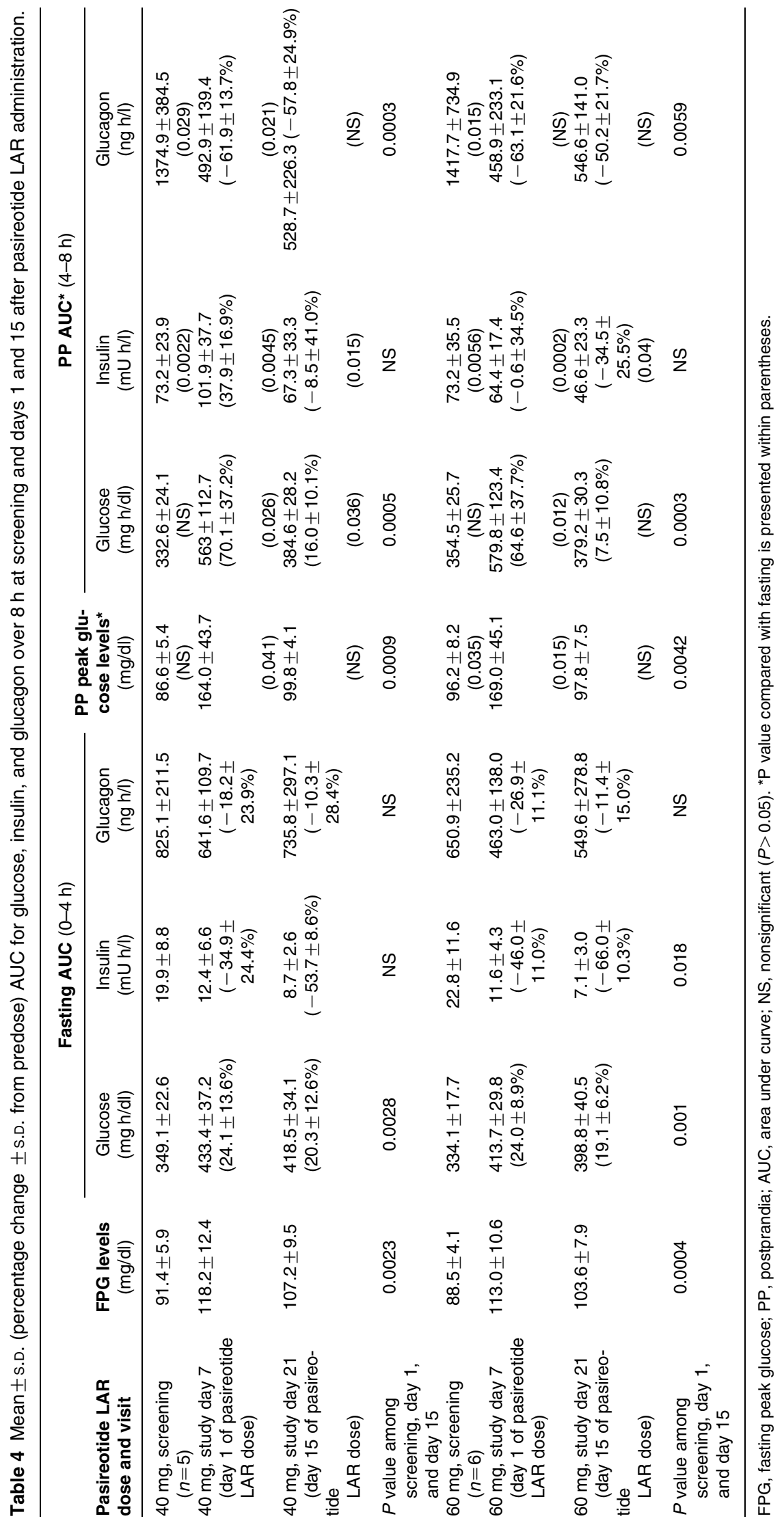


values. Postprandially, insulin AUC levels did not decrease to the extent seen in the fasting state, although the small sample size and large s.D. make interpretation of the data difficult. Glucagon levels were lower postprandially compared with fasting levels. The higher insulin levels and lower glucagon levels may be due to an incretin effect (i.e. an increase in insulin secretion after eating because of gastrointestinal secretion of glucagon-like peptide 1 and glucose-dependent insulino tropic peptide). However, incretin hormones were not measured in the current study; therefore, limited conclusions can be drawn from this study regarding the mechanism of postprandial hyperglycemia. Further studies on larger populations are warranted to address this. Changes in HbAlc were negligible, although given the short exposure time of 70 days, pasireotide was unlikely to have a discernable influence on HbAlc.

Results from a recent Phase III trial showed that treatment with twice-daily pasireotide s.c. significantly reduced cortisol levels and provided clinical benefit in patients with Cushing's disease. In this Phase III study, pasireotide had a safety profile similar to that of other somatostatin analogs (mostly transient gastrointestinal discomfort), with the exception of the degree of hyperglycemia; $\sim 70 \%$ of patients experienced a hyperglycemia-related AE (15). Although patients with Cushing's disease are predisposed to impairments in glucose homeostasis because of the effects of chronic hypercortisolism, results from that study suggest that glucose levels should be monitored during treatment with pasireotide. Hyperglycemia should be proactively managed following current guidelines. Studies on pasireotide LAR in patient populations including Cushing's disease will determine the incidence of hyperglycemia and other AEs with pasireotide LAR. In the current single-dose study on healthy volunteers, cortisol levels were not assessed.

In conclusion, the results of this study indicate that a single dose of pasireotide LAR was generally well tolerated, with mostly mild and transient AEs at doses up to $60 \mathrm{mg}$, consistent with earlier studies of pasireotide s.c. in healthy volunteers. The PK results showed a doseproportional, extended-release profile in healthy subjects. Further clinical development of pasireotide LAR is underway with studies on patient populations. Results from these studies will give further insight into the efficacy, safety, and PK of pasireotide LAR.

\section{Supplementary data}

This is linked to the online version of the paper at http://dx.doi.org/10. 1530/EJE-11-0773.

\section{Declaration of interest}

K Hu, M Ruffin, D Song, E Bouillaud, Y Wang, and J Hasskarl are employees of Novartis. H Dietrich has no conflict of interest to disclose.

\section{Funding}

This study was funded by Novartis Pharma AG.

\section{Acknowledgements}

The authors would like to thank Dr Jiangquan Zhou for major contributions to the analysis of PK data. Financial support for medical editorial assistance was provided by Novartis Pharmaceuticals Corporation. We thank Helene Darmofal for medical editorial assistance with this manuscript.

\section{References}

1 Schmid HA. Pasireotide (SOM230): development, mechanism of action and potential applications. Molecular and Cellular Endocrinology 2008286 69-74. (doi:10.1016/j.mce.2007.09.006)

2 Bruns C, Lewis I, Briner U, Meno-Tetang G \& Weckbecker G. SOM230: a novel somatostatin peptidomimetic with broad somatotropin release inhibiting factor (SRIF) receptor binding and a unique antisecretory profile. European Journal of Endocrinology 2002146 707-716. (doi:10.1530/eje.0.1460707)

3 Van Der Hoek J, Waaijers M, van Koetsveld PM, Sprij-Mooij D, Feelders RA, Schmid HA, Schoeffter P, Hoyer D, Cervia D, Taylor JE, Culler MD, Lamberts SW \& Hofland LJ. Distinct functional properties of native somatostatin receptor subtype 5 compared with subtype 2 in the regulation of ACTH release by corticotroph tumour cells. American Journal of Physiology. Endocrinology and Metabolism 2005 289 E278-E287. (doi:10.1152/ajpendo.00004.2005)

4 Batista DL, Zhang X, Gejman R, Ansell PJ, Zhou Y, Johnson SA, Swearingen B, Hedley-Whyte ET, Stratakis CA \& Klibanski A. The effects of SOM230 on cell proliferation and adrenocorticotropin secretion in human corticotroph pituitary adenomas. Journal of Clinical Endocrinology and Metabolism 200691 4482-4488. (doi:10.1210/jc.2006-1245)

5 Hofland LJ, Van Der Hoek J, Feelders R, van Aken MO, van Koetsveld PM, Waaijers M, Sprij-Mooij D, Bruns C, Weckbecker G, de Herder WW, Beckers A \& Lamberts SW. The multi-ligand somatostatin analogue SOM230 inhibits ACTH secretion by cultured human corticotroph adenomas via somatostatin receptor type 5. European Journal of Endocrinology 2005152 645-654. (doi:10.1530/eje.1.01876)

6 Murray RD, Kim K, Ren SG, Lewis I, Weckbecker G, Bruns C \& Melmed S. The novel somatostatin ligand (SOM230) regulates human and rat anterior pituitary hormone secretion. Journal of Clinical Endocrinology and Metabolism 200489 3027-3032. (doi:10.1210/jc.2003-031319)

7 Ma P, Wang Y, Van Der Hoek J, Nedelman J, Schran H, Tran L-L \& Lamberts SW. Pharmacokinetic-pharmacodynamic comparison of a novel multiligand somatostatin analog, SOM230, with octreotide in patients with acromegaly. Clinical Pharmacology and Therapeutics 200578 69-80. (doi:10.1016/j.clpt.2005.04.003)

8 Petersenn S, Unger N, Hu K, Weisshaar B, Zhang Y, Bouillaud E, Hermosillo Resendiz K, Wang Y \& Mann K. Pasireotide (SOM230), a novel multi-receptor-targeted somatostatin analogue, is well tolerated when administered as a continuous 7-day subcutaneous infusion in healthy male volunteers. Journal of Clinical Pharmacology 2012 (doi:10.1177/0091270011408727)

9 Petersenn S, Hu K, Maldonado M, Zhang Y, Lasher J, Bouillaud E, Wang Y, Mann K \& Unger N. Tolerability and dose-proportional pharmacokinetics of pasireotide administered as a single dose or two divided doses in healthy male volunteers: a single-center, open-label, ascending-dose study. Clinical Therapeutics 2012 [Epub ahead of print]. (doi:110.1016/j.clinthera.2012.01.015).

10 Golor G, Hu K, Ruffin M, Buchelt A, Bouillaud E, Wang Y \& Maldonado M. A first-in-man study to evaluate the safety, 
tolerability, and pharmacokinetics of pasireotide (SOM230), a multireceptor-targeted somatostatin analog, in healthy volunteers. Drug Design, Development and Therapy 20126 in press.

11 Shenouda M, Maldonado M, Wang Y, Bouillaud E, Hudson M, Nesheiwat D \& Hu K. An open-label dose-escalation study of oncedaily and twice-daily pasireotide in healthy volunteers: safety, tolerability, and effects on glucose, insulin, and glucagon levels. American Journal of Therapeutics 201219 in press.

12 Farrall A, Ruffin M, Wetli-Hermosillo K \& Petersenn S. Long-term treatment of acromegaly with pasireotide (SOM230): results from a phase II extension study. Endocrine Reviews 201031 (3 Suppl 1) S856 (abst OR16-5).

13 Petersenn S, Schopohl J, Barkan A, Mohideen P, Colao A, Abs R, Buchelt A, Ho Y-Y, Hu K, Farrall AJ, Melmed S \& Biller BM. Pasireotide (SOM230) demonstrates efficacy and safety in patients with acromegaly: a randomized, multicenter, phase II trial. Journal of Clinical Endocrinology and Metabolism 2010 95 2781-2789. (doi:10.1210/jc.2009-2272)

14 Boscaro M, Ludlam WH, Atkinson B, Glusman JE, Petersenn S, Reincke M, Snyder P, Tabarin A, Biller BM, Findling J, Melmed S, Darby CH, Hu K, Wang Y, Freda PU, Grossman AB, Frohman LA \&
Bertherat J. Treatment of pituitary dependent Cushing's disease with the multi-receptor ligand somatostatin analog pasireotide (SOM230): a multicenter, phase II trial. Journal of Clinical Endocrinology and Metabolism 200994 115-122. (doi:10.1210/ jc.2008-1008)

15 Colao A, Petersenn S, Newell-Price J, Findling JW, Gu F, Maldonado M, Schoenherr U, Mills D, Salgado LR \& Biller BM. A 12-month Phase 3 study of Pasireotide in Cushing's disease. New England Journal of Medicine 2012366 914-924.

16 Anthony L\& Freda PU. From somatostatin to octreotide LAR: evolution of a somatostatin analogue. Current Medical Research and Opinion 2009 25 2989-2999. (doi:10.1185/03007990903328959)

17 Freda PU. Somatostatin analogs in acromegaly. Journal of Clinical Endocrinology and Metabolism 200287 3013-3018. (doi:10. $1210 /$ jc.87.7.3013)

Received 31 August 2011

Revised version received 20 February 2012

Accepted 1 March 2012 